Article

\title{
Neutrosophic Permeable Values and Energetic Subsets with Applications in BCK/BCI-Algebras
}

\author{
Young Bae Jun ${ }^{1, *}$, Florentin Smarandache ${ }^{2}$ (D) , Seok-Zun Song ${ }^{3}$ (i) and Hashem Bordbar 4 (i) \\ 1 Department of Mathematics Education, Gyeongsang National University, Jinju 52828, Korea \\ 2 Mathematics \& Science Department, University of New Mexico, 705 Gurley Ave., Gallup, NM 87301, USA; \\ fsmarandache@gmail.com \\ 3 Department of Mathematics, Jeju National University, Jeju 63243, Korea; szsong@jejunu.ac.kr \\ 4 Postdoctoral Research Fellow, Shahid Beheshti University, Tehran 1983969411, Iran; \\ bordbar.amirh@gmail.com \\ * Correspondence: skywine@gmail.com
}

Received: 14 March 2018; Accepted: 2 May 2018; Published: 7 May 2018

\begin{abstract}
The concept of a $(\epsilon, \in)$-neutrosophic ideal is introduced, and its characterizations are established. The notions of neutrosophic permeable values are introduced, and related properties are investigated. Conditions for the neutrosophic level sets to be energetic, right stable, and right vanished are discussed. Relations between neutrosophic permeable $S$ - and $I$-values are considered.
\end{abstract}

Keywords: $(\in, \in)$-neutrosophic subalgebra; $(\in, \in)$-neutrosophic ideal; neutrosophic (anti-)permeable $S$-value; neutrosophic (anti-)permeable I-value; $S$-energetic set; I-energetic set

MSC: 06F35; 03G25; 08A72

\section{Introduction}

The notion of neutrosophic set (NS) theory developed by Smarandache (see [1,2]) is a more general platform that extends the concepts of classic and fuzzy sets, intuitionistic fuzzy sets, and interval-valued (intuitionistic) fuzzy sets and that is applied to various parts: pattern recognition, medical diagnosis, decision-making problems, and so on (see [3-6]). Smarandache [2] mentioned that a cloud is a NS because its borders are ambiguous and because each element (water drop) belongs with a neutrosophic probability to the set (e.g., there are types of separated water drops around a compact mass of water drops, such that we do not know how to consider them: in or out of the cloud). Additionally, we are not sure where the cloud ends nor where it begins, and neither whether some elements are or are not in the set. This is why the percentage of indeterminacy is required and the neutrosophic probability (using subsets-not numbers-as components) should be used for better modeling: it is a more organic, smooth, and particularly accurate estimation. Indeterminacy is the zone of ignorance of a proposition's value, between truth and falsehood.

Algebraic structures play an important role in mathematics with wide-ranging applications in several disciplines such as coding theory, information sciences, computer sciences, control engineering, theoretical physics, and so on. NS theory is also applied to several algebraic structures. In particular, Jun et al. applied it to $B C K / B C I$-algebras (see [7-12]). Jun et al. [8] introduced the notions of energetic subsets, right vanished subsets, right stable subsets, and (anti-)permeable values in $B C K / B C I$-algebras and investigated relations between these sets.

In this paper, we introduce the notions of neutrosophic permeable $S$-values, neutrosophic permeable $I$-values, $(\in, \in)$-neutrosophic ideals, neutrosophic anti-permeable $S$-values, and neutrosophic anti-permeable $I$-values, which are motivated by the idea of subalgebras 
(i.e., $S$-values) and ideals (i.e., I-values), and investigate their properties. We consider characterizations of $(\in, \in)$-neutrosophic ideals. We discuss conditions for the lower (upper) neutrosophic $\epsilon_{\Phi}$-subsets to be $S$ - and $I$-energetic. We provide conditions for a triple $(\alpha, \beta, \gamma)$ of numbers to be a neutrosophic (anti-)permeable $S$ - or $I$-value. We consider conditions for the upper (lower) neutrosophic $\in_{\Phi}$-subsets to be right stable (right vanished) subsets. We establish relations between neutrosophic (anti-)permeable $S$ - and $I$-values.

\section{Preliminaries}

An algebra $(X ; *, 0)$ of type $(2,0)$ is called a BCI-algebra if it satisfies the following conditions:

(I) $\quad(\forall x, y, z \in X)(((x * y) *(x * z)) *(z * y)=0)$;

(II) $\quad(\forall x, y \in X)((x *(x * y)) * y=0)$;

(III) $(\forall x \in X)(x * x=0)$;

(IV) $(\forall x, y \in X)(x * y=0, y * x=0 \Rightarrow x=y)$.

If a $B C I$-algebra $X$ satisfies the following identity:

(V) $(\forall x \in X)(0 * x=0)$,

then $X$ is called a $B C K$-algebra. Any $B C K / B C I$-algebra $X$ satisfies the following conditions:

$$
\begin{aligned}
& (\forall x \in X)(x * 0=x), \\
& (\forall x, y, z \in X)(x \leq y \Rightarrow x * z \leq y * z, z * y \leq z * x), \\
& (\forall x, y, z \in X)((x * y) * z=(x * z) * y), \\
& (\forall x, y, z \in X)((x * z) *(y * z) \leq x * y),
\end{aligned}
$$

where $x \leq y$ if and only if $x * y=0$. A nonempty subset $S$ of a $B C K / B C I$-algebra $X$ is called a subalgebra of $X$ if $x * y \in S$ for all $x, y \in S$. A subset $I$ of a $B C K / B C I$-algebra $X$ is called an ideal of $X$ if it satisfies the following:

$$
\begin{aligned}
& 0 \in I, \\
& (\forall x, y \in X)(x * y \in I, y \in I \rightarrow x \in I) .
\end{aligned}
$$

We refer the reader to the books [13] and [14] for further information regarding $B C K / B C I$-algebras.

For any family $\left\{a_{i} \mid i \in \Lambda\right\}$ of real numbers, we define

$$
\bigvee\left\{a_{i} \mid i \in \Lambda\right\}=\sup \left\{a_{i} \mid i \in \Lambda\right\}
$$

and

$$
\bigwedge\left\{a_{i} \mid i \in \Lambda\right\}=\inf \left\{a_{i} \mid i \in \Lambda\right\}
$$

If $\Lambda=\{1,2\}$, we also use $a_{1} \vee a_{2}$ and $a_{1} \wedge a_{2}$ instead of $\bigvee\left\{a_{i} \mid i \in\{1,2\}\right\}$ and $\wedge\left\{a_{i} \mid i \in\{1,2\}\right\}$, respectively.

We let $X$ be a nonempty set. A NS in $X$ (see [1]) is a structure of the form

$$
A:=\left\{\left\langle x ; A_{T}(x), A_{I}(x), A_{F}(x)\right\rangle \mid x \in X\right\},
$$

where $A_{T}: X \rightarrow[0,1]$ is a truth membership function, $A_{I}: X \rightarrow[0,1]$ is an indeterminate membership function, and $A_{F}: X \rightarrow[0,1]$ is a false membership function. For the sake of simplicity, we use the $\operatorname{symbol} A=\left(A_{T}, A_{I}, A_{F}\right)$ for the NS

$$
A:=\left\{\left\langle x ; A_{T}(x), A_{I}(x), A_{F}(x)\right\rangle \mid x \in X\right\}
$$


A subset $A$ of a $B C K / B C I$-algebra $X$ is said to be $S$-energetic (see [8]) if it satisfies

$$
(\forall x, y \in X)(x * y \in A \Rightarrow\{x, y\} \cap A \neq \varnothing) .
$$

A subset $A$ of a $B C K / B C I$-algebra $X$ is said to be I-energetic (see [8]) if it satisfies

$$
(\forall x, y \in X)(y \in A \Rightarrow\{x, y * x\} \cap A \neq \varnothing) .
$$

A subset $A$ of a $B C K / B C I$-algebra $X$ is said to be right vanished (see [8]) if it satisfies

$$
(\forall x, y \in X)(x * y \in A \Rightarrow x \in A) .
$$

A subset $A$ of a $B C K / B C I$-algebra $X$ is said to be right stable (see [8]) if $A * X:=\{a * x \mid a \in$ $A, x \in X\} \subseteq A$.

\section{Neutrosophic Permeable Values}

Given a NS $A=\left(A_{T}, A_{I}, A_{F}\right)$ in a set $X, \alpha, \beta \in(0,1]$ and $\gamma \in[0,1)$, we consider the following sets:

$$
\begin{aligned}
& U_{T}^{\in}(A ; \alpha)=\left\{x \in X \mid A_{T}(x) \geq \alpha\right\}, U_{T}^{\in}(A ; \alpha)^{*}=\left\{x \in X \mid A_{T}(x)>\alpha\right\}, \\
& U_{I}^{\epsilon}(A ; \beta)=\left\{x \in X \mid A_{I}(x) \geq \beta\right\}, U_{I}^{\epsilon}(A ; \beta)^{*}=\left\{x \in X \mid A_{I}(x)>\beta\right\}, \\
& U_{F}^{\in}(A ; \gamma)=\left\{x \in X \mid A_{F}(x) \leq \gamma\right\}, U_{F}^{\in}(A ; \gamma)^{*}=\left\{x \in X \mid A_{F}(x)<\gamma\right\}, \\
& L_{T}^{\epsilon}(A ; \alpha)=\left\{x \in X \mid A_{T}(x) \leq \alpha\right\}, L_{T}^{\in}(A ; \alpha)^{*}=\left\{x \in X \mid A_{T}(x)<\alpha\right\}, \\
& L_{I}^{\epsilon}(A ; \beta)=\left\{x \in X \mid A_{I}(x) \leq \beta\right\}, L_{I}^{\epsilon}(A ; \beta)^{*}=\left\{x \in X \mid A_{I}(x)<\beta\right\}, \\
& L_{F}^{\epsilon}(A ; \gamma)=\left\{x \in X \mid A_{F}(x) \geq \gamma\right\}, L_{F}^{\in}(A ; \gamma)^{*}=\left\{x \in X \mid A_{F}(x)>\gamma\right\},
\end{aligned}
$$

We say $U_{T}^{\epsilon}(A ; \alpha), U_{I}^{\epsilon}(A ; \beta)$, and $U_{F}^{\epsilon}(A ; \gamma)$ are upper neutrosophic $\epsilon_{\Phi}$-subsets of $X$, and $L_{T}^{\epsilon}(A ; \alpha)$, $L_{I}^{\in}(A ; \beta)$, and $L_{F}^{\in}(A ; \gamma)$ are lower neutrosophic $\in_{\Phi}$-subsets of $X$, where $\Phi \in\{T, I, F\}$. We say $U_{T}^{\in}(A ; \alpha)^{*}$,

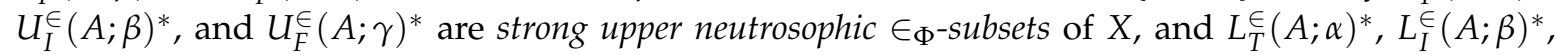
and $L_{F}^{\in}(A ; \gamma)^{*}$ are strong lower neutrosophic $\in_{\Phi}$-subsets of $X$, where $\Phi \in\{T, I, F\}$.

Definition 1 ([7]). A NS $A=\left(A_{T}, A_{I}, A_{F}\right)$ in a $B C K / B C I$-algebra $X$ is called an $(\in, \in)$ neutrosophic subalgebra of $X$ if the following assertions are valid:

$$
\begin{aligned}
& x \in U_{T}^{\epsilon}\left(A ; \alpha_{x}\right), y \in U_{T}^{\epsilon}\left(A ; \alpha_{y}\right) \Rightarrow x * y \in U_{T}^{\in}\left(A ; \alpha_{x} \wedge \alpha_{y}\right), \\
& x \in U_{I}^{\epsilon}\left(A ; \beta_{x}\right), y \in U_{I}^{\epsilon}\left(A ; \beta_{y}\right) \Rightarrow x * y \in U_{I}^{\epsilon}\left(A ; \beta_{x} \wedge \beta_{y}\right), \\
& x \in U_{F}^{\epsilon}\left(A ; \gamma_{x}\right), y \in U_{F}^{\epsilon}\left(A ; \gamma_{y}\right) \Rightarrow x * y \in U_{F}^{\in}\left(A ; \gamma_{x} \vee \gamma_{y}\right),
\end{aligned}
$$

for all $x, y \in X, \alpha_{x}, \alpha_{y}, \beta_{x}, \beta_{y} \in(0,1]$ and $\gamma_{x}, \gamma_{y} \in[0,1)$.

Lemma 1 ([7]). A NS $A=\left(A_{T}, A_{I}, A_{F}\right)$ in a $B C K / B C I$-algebra $X$ is an $(\epsilon, \in)$-neutrosophic subalgebra of $X$ if and only if $A=\left(A_{T}, A_{I}, A_{F}\right)$ satisfies

$$
(\forall x, y \in X)\left(\begin{array}{l}
A_{T}(x * y) \geq A_{T}(x) \wedge A_{T}(y) \\
A_{I}(x * y) \geq A_{I}(x) \wedge A_{I}(y) \\
A_{F}(x * y) \leq A_{F}(x) \vee A_{F}(y)
\end{array}\right) .
$$

Proposition 1. Every $(\in, \in)$-neutrosophic subalgebra $A=\left(A_{T}, A_{I}, A_{F}\right)$ of a $B C K / B C I$-algebra X satisfies

$$
(\forall x \in X)\left(A_{T}(0) \geq A_{T}(x), A_{I}(0) \geq A_{I}(x), A_{F}(0) \leq A_{F}(x)\right) .
$$


Proof. Straightforward.

Theorem 1. If $A=\left(A_{T}, A_{I}, A_{F}\right)$ is an $(\in, \in)$-neutrosophic subalgebra of a BCK/BCI-algebra $X$, then the

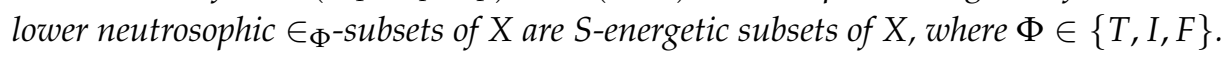

Proof. Let $x, y \in X$ and $\alpha \in(0,1]$ be such that $x * y \in L_{T}^{\in}(A ; \alpha)$. Then

$$
\alpha \geq A_{T}(x * y) \geq A_{T}(x) \wedge A_{T}(y)
$$

and thus $A_{T}(x) \leq \alpha$ or $A_{T}(y) \leq \alpha$; that is, $x \in L_{T}^{\in}(A ; \alpha)$ or $y \in L_{T}^{\in}(A ; \alpha)$. Thus $\{x, y\} \cap L_{T}^{\in}(A ; \alpha) \neq \varnothing$. Therefore $L_{T}^{\in}(A ; \alpha)$ is an $S$-energetic subset of $X$. Similarly, we can verify that $L_{I}^{\in}(A ; \beta)$ is an $S$-energetic subset of $X$. We let $x, y \in X$ and $\gamma \in[0,1)$ be such that $x * y \in L_{F}^{\in}(A ; \gamma)$. Then

$$
\gamma \leq A_{F}(x * y) \leq A_{F}(x) \vee A_{F}(y)
$$

It follows that $A_{F}(x) \geq \gamma$ or $A_{F}(y) \geq \gamma$; that is, $x \in L_{F}^{\in}(A ; \gamma)$ or $y \in L_{F}^{\in}(A ; \gamma)$. Hence $\{x, y\} \cap$ $L_{F}^{\in}(A ; \gamma) \neq \varnothing$, and therefore $L_{F}^{\in}(A ; \gamma)$ is an $S$-energetic subset of $X$.

Corollary 1. If $A=\left(A_{T}, A_{I}, A_{F}\right)$ is an $(\in, \in)$-neutrosophic subalgebra of a BCK/BCI-algebra $X$, then the strong lower neutrosophic $\in_{\Phi}$-subsets of $X$ are $S$-energetic subsets of $X$, where $\Phi \in\{T, I, F\}$.

Proof. Straightforward.

The converse of Theorem 1 is not true, as seen in the following example.

Example 1. Consider a $B C K$-algebra $X=\{0,1,2,3,4\}$ with the binary operation $*$ that is given in Table 1 (see [14]).

Table 1. Cayley table for the binary operation " $*$ ".

\begin{tabular}{llllll}
\hline * & $\mathbf{0}$ & $\mathbf{1}$ & $\mathbf{2}$ & $\mathbf{3}$ & $\mathbf{4}$ \\
\hline 0 & 0 & 0 & 0 & 0 & 0 \\
1 & 1 & 0 & 0 & 0 & 0 \\
2 & 2 & 1 & 0 & 0 & 1 \\
3 & 3 & 2 & 1 & 0 & 2 \\
4 & 4 & 1 & 1 & 1 & 0 \\
\hline
\end{tabular}

Let $A=\left(A_{T}, A_{I}, A_{F}\right)$ be a NS in $X$ that is given in Table 2.

Table 2. Tabulation representation of $A=\left(A_{T}, A_{I}, A_{F}\right)$.

\begin{tabular}{cccc}
\hline $\boldsymbol{X}$ & $\boldsymbol{A}_{\boldsymbol{T}}(\boldsymbol{x})$ & $\boldsymbol{A}_{\boldsymbol{I}}(\boldsymbol{x})$ & $\boldsymbol{A}_{\boldsymbol{F}}(\boldsymbol{x})$ \\
\hline 0 & 0.6 & 0.8 & 0.2 \\
1 & 0.4 & 0.5 & 0.7 \\
2 & 0.4 & 0.5 & 0.6 \\
3 & 0.4 & 0.5 & 0.5 \\
4 & 0.7 & 0.8 & 0.2 \\
\hline
\end{tabular}

If $\alpha \in[0.4,0.6), \beta \in[0.5,0.8)$, and $\gamma \in(0.2,0.5]$, then $L_{T}^{\in}(A ; \alpha)=\{1,2,3\}, L_{I}^{\in}(A ; \beta)=\{1,2,3\}$, and $L_{F}^{\in}(A ; \gamma)=\{1,2,3\}$ are $S$-energetic subsets of $X$. Because

$$
A_{T}(4 * 4)=A_{T}(0)=0.6 \ngtr 0.7=A_{T}(4) \wedge A_{T}(4)
$$


and/or

$$
A_{F}(3 * 2)=A_{F}(1)=0.7 \not \leq 0.6=A_{F}(3) \vee A_{F}(2) \text {, }
$$

it follows from Lemma 1 that $A=\left(A_{T}, A_{I}, A_{F}\right)$ is not an $(\in, \in)$-neutrosophic subalgebra of $X$.

Definition 2. Let $A=\left(A_{T}, A_{I}, A_{F}\right)$ be a NS in a BCK/BCI-algebra $X$ and $(\alpha, \beta, \gamma) \in \Lambda_{T} \times \Lambda_{I} \times \Lambda_{F}$, where $\Lambda_{T}, \Lambda_{I}$, and $\Lambda_{F}$ are subsets of $[0,1]$. Then $(\alpha, \beta, \gamma)$ is called a neutrosophic permeable $S$-value for $A=\left(A_{T}, A_{I}, A_{F}\right)$ if the following assertion is valid:

$$
(\forall x, y \in X)\left(\begin{array}{l}
x * y \in U_{T}^{\in}(A ; \alpha) \Rightarrow A_{T}(x) \vee A_{T}(y) \geq \alpha, \\
x * y \in U_{I}^{\in}(A ; \beta) \Rightarrow A_{I}(x) \vee A_{I}(y) \geq \beta, \\
x * y \in U_{F}^{\in}(A ; \gamma) \Rightarrow A_{F}(x) \wedge A_{F}(y) \leq \gamma
\end{array}\right)
$$

Example 2. Let $X=\{0,1,2,3,4\}$ be a set with the binary operation $*$ that is given in Table 3 .

Table 3. Cayley table for the binary operation " $*$ ".

\begin{tabular}{llllll}
\hline * & $\mathbf{0}$ & $\mathbf{1}$ & $\mathbf{2}$ & $\mathbf{3}$ & $\mathbf{4}$ \\
\hline 0 & 0 & 0 & 0 & 0 & 0 \\
1 & 1 & 0 & 1 & 1 & 0 \\
2 & 2 & 2 & 0 & 2 & 0 \\
3 & 3 & 3 & 3 & 0 & 3 \\
4 & 4 & 4 & 4 & 4 & 0 \\
\hline
\end{tabular}

Then $(X, *, 0)$ is a BCK-algebra (see [14]). Let $A=\left(A_{T}, A_{I}, A_{F}\right)$ be a NS in X that is given in Table 4 .

Table 4. Tabulation representation of $A=\left(A_{T}, A_{I}, A_{F}\right)$.

\begin{tabular}{cccc}
\hline $\boldsymbol{X}$ & $A_{T}(\boldsymbol{x})$ & $A_{\boldsymbol{I}}(\boldsymbol{x})$ & $A_{\boldsymbol{F}}(\boldsymbol{x})$ \\
\hline 0 & 0.2 & 0.3 & 0.7 \\
1 & 0.6 & 0.4 & 0.6 \\
2 & 0.5 & 0.3 & 0.4 \\
3 & 0.4 & 0.8 & 0.5 \\
4 & 0.7 & 0.6 & 0.2 \\
\hline
\end{tabular}

It is routine to verify that $(\alpha, \beta, \gamma) \in(0,2,1] \times(0.3,1] \times[0,0.7)$ is a neutrosophic permeable S-value for $A=\left(A_{T}, A_{I}, A_{F}\right)$.

Theorem 2. Let $A=\left(A_{T}, A_{I}, A_{F}\right)$ be a $N S$ in a $B C K / B C I$-algebra $X$ and $(\alpha, \beta, \gamma) \in \Lambda_{T} \times \Lambda_{I} \times \Lambda_{F}$, where $\Lambda_{T}, \Lambda_{I}$, and $\Lambda_{F}$ are subsets of $[0,1]$. If $A=\left(A_{T}, A_{I}, A_{F}\right)$ satisfies the following condition:

$$
(\forall x, y \in X)\left(\begin{array}{l}
A_{T}(x * y) \leq A_{T}(x) \vee A_{T}(y) \\
A_{I}(x * y) \leq A_{I}(x) \vee A_{I}(y) \\
A_{F}(x * y) \geq A_{F}(x) \wedge A_{F}(y)
\end{array}\right)
$$

then $(\alpha, \beta, \gamma)$ is a neutrosophic permeable S-value for $A=\left(A_{T}, A_{I}, A_{F}\right)$.

Proof. Let $x, y \in X$ be such that $x * y \in U_{T}^{\in}(A ; \alpha)$. Then

$$
\alpha \leq A_{T}(x * y) \leq A_{T}(x) \vee A_{T}(y)
$$


Similarly, if $x * y \in U_{I}^{\in}(A ; \beta)$ for $x, y \in X$, then $A_{I}(x) \vee A_{I}(y) \geq \beta$. Now, let $a, b \in X$ be such that $a * b \in U_{F}^{\in}(A ; \gamma)$. Then

$$
\gamma \geq A_{F}(a * b) \geq A_{F}(a) \wedge A_{F}(b)
$$

Therefore $(\alpha, \beta, \gamma)$ is a neutrosophic permeable $S$-value for $A=\left(A_{T}, A_{I}, A_{F}\right)$.

Theorem 3. Let $A=\left(A_{T}, A_{I}, A_{F}\right)$ be a NS in a $B C K$-algebra $X$ and $(\alpha, \beta, \gamma) \in \Lambda_{T} \times \Lambda_{I} \times \Lambda_{F}$, where $\Lambda_{T}$, $\Lambda_{I}$, and $\Lambda_{F}$ are subsets of $[0,1]$. If $A=\left(A_{T}, A_{I}, A_{F}\right)$ satisfies the following conditions:

$$
(\forall x \in X)\left(A_{T}(0) \leq A_{T}(x), A_{I}(0) \leq A_{I}(x), A_{F}(0) \geq A_{F}(x)\right)
$$

and

$$
(\forall x, y \in X)\left(\begin{array}{l}
A_{T}(x) \leq A_{T}(x * y) \vee A_{T}(y) \\
A_{I}(x) \leq A_{I}(x * y) \vee A_{I}(y) \\
A_{F}(x) \geq A_{F}(x * y) \wedge A_{F}(y)
\end{array}\right)
$$

then $(\alpha, \beta, \gamma)$ is a neutrosophic permeable S-value for $A=\left(A_{T}, A_{I}, A_{F}\right)$.

Proof. Let $x, y, a, b, u, v \in X$ be such that $x * y \in U_{T}^{\in}(A ; \alpha), a * b \in U_{I}^{\in}(A ; \beta)$, and $u * v \in U_{F}^{\in}(A ; \gamma)$. Then

$$
\begin{aligned}
\alpha & \leq A_{T}(x * y) \leq A_{T}((x * y) * x) \vee A_{T}(x) \\
& =A_{T}((x * x) * y) \vee A_{T}(x)=A_{T}(0 * y) \vee A_{T}(x) \\
& =A_{T}(0) \vee A_{T}(x)=A_{T}(x), \\
\beta & \leq A_{I}(a * b) \leq A_{I}((a * b) * a) \vee A_{I}(a) \\
& =A_{I}((a * a) * b) \vee A_{I}(a)=A_{I}(0 * b) \vee A_{I}(a) \\
& =A_{I}(0) \vee A_{I}(a)=A_{I}(a),
\end{aligned}
$$

and

$$
\begin{aligned}
\gamma & \geq A_{F}(u * v) \geq A_{F}((u * v) * u) \wedge A_{F}(u) \\
& =A_{F}((u * u) * v) \wedge A_{F}(u)=A_{F}(0 * v) \wedge A_{F}(v) \\
& =A_{F}(0) \wedge A_{F}(v)=A_{F}(v)
\end{aligned}
$$

by Equations (3), (V), (15), and (16). It follows that

$$
\begin{aligned}
& A_{T}(x) \vee A_{T}(y) \geq A_{T}(x) \geq \alpha, \\
& A_{I}(a) \vee A_{I}(b) \geq A_{I}(a) \geq \beta, \\
& A_{F}(u) \wedge A_{F}(v) \leq A_{F}(u) \leq \gamma .
\end{aligned}
$$

Therefore $(\alpha, \beta, \gamma)$ is a neutrosophic permeable $S$-value for $A=\left(A_{T}, A_{I}, A_{F}\right)$.

Theorem 4. Let $A=\left(A_{T}, A_{I}, A_{F}\right)$ be a NS in a BCK/BCI-algebra $X$ and $(\alpha, \beta, \gamma) \in \Lambda_{T} \times \Lambda_{I} \times \Lambda_{F}$, where $\Lambda_{T}, \Lambda_{I}$, and $\Lambda_{F}$ are subsets of $[0,1]$. If $(\alpha, \beta, \gamma)$ is a neutrosophic permeable $S$-value for $A=\left(A_{T}, A_{I}\right.$,

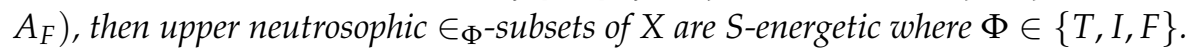


Proof. Let $x, y, a, b, u, v \in X$ be such that $x * y \in U_{T}^{\in}(A ; \alpha), a * b \in U_{I}^{\in}(A ; \beta)$, and $u * v \in U_{F}^{\in}(A ; \gamma)$. Using Equation (13), we have $A_{T}(x) \vee A_{T}(y) \geq \alpha, A_{I}(a) \vee A_{I}(b) \geq \beta$, and $A_{F}(u) \wedge A_{F}(v) \leq \gamma$. It follows that

$$
\begin{aligned}
& A_{T}(x) \geq \alpha \text { or } A_{T}(y) \geq \alpha, \text { that is, } x \in U_{T}^{\in}(A ; \alpha) \text { or } y \in U_{T}^{\in}(A ; \alpha) \\
& A_{I}(a) \geq \beta \text { or } A_{I}(b) \geq \beta, \text { that is, } a \in U_{I}^{\in}(A ; \beta) \text { or } b \in U_{I}^{\in}(A ; \beta)
\end{aligned}
$$

and

$$
A_{F}(u) \leq \gamma \text { or } A_{F}(v) \leq \gamma \text {, that is, } u \in U_{F}^{\in}(A ; \gamma) \text { or } v \in U_{F}^{\in}(A ; \gamma)
$$

Hence $\{x, y\} \cap U_{T}^{\in}(A ; \alpha) \neq \varnothing,\{a, b\} \cap U_{I}^{\in}(A ; \beta) \neq \varnothing$, and $\{u, v\} \cap U_{F}^{\in}(A ; \gamma) \neq \varnothing$. Therefore $U_{T}^{\in}(A ; \alpha), U_{I}^{\in}(A ; \beta)$, and $U_{F}^{\in}(A ; \gamma)$ are $S$-energetic subsets of $X$.

Definition 3. Let $A=\left(A_{T}, A_{I}, A_{F}\right)$ be a NS in a BCK/BCI-algebra X and $(\alpha, \beta, \gamma) \in \Lambda_{T} \times \Lambda_{I} \times \Lambda_{F}$, where $\Lambda_{T}, \Lambda_{I}$, and $\Lambda_{F}$ are subsets of $[0,1]$. Then $(\alpha, \beta, \gamma)$ is called a neutrosophic anti-permeable $S$-value for $A=\left(A_{T}, A_{I}, A_{F}\right)$ if the following assertion is valid:

$$
(\forall x, y \in X)\left(\begin{array}{l}
x * y \in L_{T}^{\in}(A ; \alpha) \Rightarrow A_{T}(x) \wedge A_{T}(y) \leq \alpha \\
x * y \in L_{I}^{\in}(A ; \beta) \Rightarrow A_{I}(x) \wedge A_{I}(y) \leq \beta \\
x * y \in L_{F}^{\in}(A ; \gamma) \Rightarrow A_{F}(x) \vee A_{F}(y) \geq \gamma
\end{array}\right)
$$

Example 3. Let $X=\{0,1,2,3,4\}$ be a set with the binary operation $*$ that is given in Table 5 .

Table 5. Cayley table for the binary operation "*”.

\begin{tabular}{llllll}
\hline * & $\mathbf{0}$ & $\mathbf{1}$ & $\mathbf{2}$ & $\mathbf{3}$ & $\mathbf{4}$ \\
\hline 0 & 0 & 0 & 0 & 0 & 0 \\
1 & 1 & 0 & 0 & 1 & 0 \\
2 & 2 & 1 & 0 & 2 & 0 \\
3 & 3 & 3 & 3 & 0 & 3 \\
4 & 4 & 4 & 4 & 4 & 0 \\
\hline
\end{tabular}

Then $(X, *, 0)$ is a BCK-algebra (see [14]). Let $A=\left(A_{T}, A_{I}, A_{F}\right)$ be a NS in X that is given in Table 6.

Table 6. Tabulation representation of $A=\left(A_{T}, A_{I}, A_{F}\right)$.

\begin{tabular}{cccc}
\hline $\boldsymbol{X}$ & $\boldsymbol{A}_{\boldsymbol{T}}(\boldsymbol{x})$ & $\boldsymbol{A}_{\boldsymbol{I}}(\boldsymbol{x})$ & $\boldsymbol{A}_{\boldsymbol{F}}(\boldsymbol{x})$ \\
\hline 0 & 0.7 & 0.6 & 0.4 \\
1 & 0.4 & 0.5 & 0.6 \\
2 & 0.4 & 0.5 & 0.6 \\
3 & 0.5 & 0.2 & 0.7 \\
4 & 0.3 & 0.3 & 0.9 \\
\hline
\end{tabular}

It is routine to verify that $(\alpha, \beta, \gamma) \in(0.3,1] \times(0.2,1] \times[0,0.9)$ is a neutrosophic anti-permeable S-value for $A=\left(A_{T}, A_{I}, A_{F}\right)$.

Theorem 5. Let $A=\left(A_{T}, A_{I}, A_{F}\right)$ be a NS in a $B C K / B C I$-algebra $X$ and $(\alpha, \beta, \gamma) \in \Lambda_{T} \times \Lambda_{I} \times \Lambda_{F}$, where $\Lambda_{T}, \Lambda_{I}$, and $\Lambda_{F}$ are subsets of $[0,1]$. If $A=\left(A_{T}, A_{I}, A_{F}\right)$ is an $(\in, \in)$-neutrosophic subalgebra of $X$, then $(\alpha, \beta, \gamma)$ is a neutrosophic anti-permeable $S$-value for $A=\left(A_{T}, A_{I}, A_{F}\right)$. 
Proof. Let $x, y, a, b, u, v \in X$ be such that $x * y \in L_{T}^{\in}(A ; \alpha), a * b \in L_{I}^{\in}(A ; \beta)$, and $u * v \in L_{F}^{\in}(A ; \gamma)$. Using Lemma 1 , we have

$$
\begin{aligned}
& A_{T}(x) \wedge A_{T}(y) \leq A_{T}(x * y) \leq \alpha, \\
& A_{I}(a) \wedge A_{I}(b) \leq A_{I}(a * b) \leq \beta, \\
& A_{F}(u) \vee A_{F}(v) \geq A_{F}(u * v) \geq \gamma,
\end{aligned}
$$

and thus $(\alpha, \beta, \gamma)$ is a neutrosophic anti-permeable $S$-value for $A=\left(A_{T}, A_{I}, A_{F}\right)$.

Theorem 6. Let $A=\left(A_{T}, A_{I}, A_{F}\right)$ be a NS in a BCK/BCI-algebra $X$ and $(\alpha, \beta, \gamma) \in \Lambda_{T} \times \Lambda_{I} \times \Lambda_{F}$, where $\Lambda_{T}, \Lambda_{I}$, and $\Lambda_{F}$ are subsets of $[0,1]$. If $(\alpha, \beta, \gamma)$ is a neutrosophic anti-permeable $S$-value for $A=\left(A_{T}\right.$, $\left.A_{I}, A_{F}\right)$, then lower neutrosophic $\in_{\Phi}$-subsets of $X$ are S-energetic where $\Phi \in\{T, I, F\}$.

Proof. Let $x, y, a, b, u, v \in X$ be such that $x * y \in L_{T}^{\in}(A ; \alpha), a * b \in L_{I}^{\in}(A ; \beta)$, and $u * v \in L_{F}^{\in}(A ; \gamma)$. Using Equation (17), we have $A_{T}(x) \wedge A_{T}(y) \leq \alpha, A_{I}(a) \wedge A_{I}(b) \leq \beta$, and $A_{F}(u) \vee A_{F}(v) \geq \gamma$, which imply that

$$
\begin{aligned}
& A_{T}(x) \leq \alpha \text { or } A_{T}(y) \leq \alpha, \text { that is, } x \in L_{T}^{\in}(A ; \alpha) \text { or } y \in L_{T}^{\in}(A ; \alpha) ; \\
& A_{I}(a) \leq \beta \text { or } A_{I}(b) \leq \beta, \text { that is, } a \in L_{I}^{\in}(A ; \beta) \text { or } b \in L_{I}^{\in}(A ; \beta) ;
\end{aligned}
$$

and

$$
A_{F}(u) \geq \gamma \text { or } A_{F}(v) \geq \gamma \text {, that is, } u \in L_{F}^{\epsilon}(A ; \gamma) \text { or } v \in L_{F}^{\epsilon}(A ; \gamma) \text {. }
$$

Hence $\{x, y\} \cap L_{T}^{\in}(A ; \alpha) \neq \varnothing,\{a, b\} \cap L_{I}^{\in}(A ; \beta) \neq \varnothing$, and $\{u, v\} \cap L_{F}^{\in}(A ; \gamma) \neq \varnothing$. Therefore $L_{T}^{\in}(A ; \alpha), L_{I}^{\in}(A ; \beta)$, and $L_{F}^{\in}(A ; \gamma)$ are $S$-energetic subsets of $X$.

Definition 4. A NS $A=\left(A_{T}, A_{I}, A_{F}\right)$ in a $B C K / B C I$-algebra $X$ is called an $(\in, \in)$-neutrosophic ideal of $X$ if the following assertions are valid:

$$
\begin{aligned}
& (\forall x \in X)\left(\begin{array}{l}
x \in U_{T}^{\in}(A ; \alpha) \Rightarrow 0 \in U_{T}^{\in}(A ; \alpha) \\
x \in U_{I}^{\epsilon}(A ; \beta) \Rightarrow 0 \in U_{I}^{\epsilon}(A ; \beta) \\
x \in U_{F}^{\epsilon}(A ; \gamma) \Rightarrow 0 \in U_{F}^{\epsilon}(A ; \gamma)
\end{array}\right), \\
& (\forall x, y \in X)\left(\begin{array}{l}
x * y \in U_{T}^{\epsilon}\left(A ; \alpha_{x}\right), y \in U_{T}^{\epsilon}\left(A ; \alpha_{y}\right) \Rightarrow x \in U_{T}^{\in}\left(A ; \alpha_{x} \wedge \alpha_{y}\right) \\
x * y \in U_{I}^{\epsilon}\left(A ; \beta_{x}\right), y \in U_{I}^{\epsilon}\left(A ; \beta_{y}\right) \Rightarrow x \in U_{I}^{\epsilon}\left(A ; \beta_{x} \wedge \beta_{y}\right) \\
x * y \in U_{F}^{\epsilon}\left(A ; \gamma_{x}\right), y \in U_{F}^{\epsilon}\left(A ; \gamma_{y}\right) \Rightarrow x \in U_{F}^{\epsilon}\left(A ; \gamma_{x} \vee \gamma_{y}\right)
\end{array}\right),
\end{aligned}
$$

for all $\alpha, \beta, \alpha_{x}, \alpha_{y}, \beta_{x}, \beta_{y} \in(0,1]$ and $\gamma, \gamma_{x}, \gamma_{y} \in[0,1)$.

Theorem 7. $A$ NS $A=\left(A_{T}, A_{I}, A_{F}\right)$ in a $B C K / B C I$-algebra $X$ is an $(\epsilon, \in)$-neutrosophic ideal of $X$ if and only if $A=\left(A_{T}, A_{I}, A_{F}\right)$ satisfies

$$
(\forall x, y \in X)\left(\begin{array}{l}
A_{T}(0) \geq A_{T}(x) \geq A_{T}(x * y) \wedge A_{T}(y) \\
A_{I}(0) \geq A_{I}(x) \geq A_{I}(x * y) \wedge A_{I}(y) \\
A_{F}(0) \leq A_{F}(x) \leq A_{F}(x * y) \vee A_{F}(y)
\end{array}\right) .
$$

Proof. Assume that Equation (20) is valid, and let $x \in U_{T}^{\in}(A ; \alpha), a \in U_{I}^{\epsilon}(A ; \beta)$, and $u \in U_{F}^{\in}(A ; \gamma)$ for any $x, a, u \in X, \alpha, \beta \in(0,1]$ and $\gamma \in[0,1)$. Then $A_{T}(0) \geq A_{T}(x) \geq \alpha, A_{I}(0) \geq A_{I}(a) \geq \beta$, and $A_{F}(0) \leq A_{F}(u) \leq \gamma$. Hence $0 \in U_{T}^{\epsilon}(A ; \alpha), 0 \in U_{I}^{\epsilon}(A ; \beta)$, and $0 \in U_{F}^{\in}(A ; \gamma)$, and thus Equation (18) is valid. Let $x, y, a, b, u, v \in X$ be such that $x * y \in U_{T}^{\in}\left(A ; \alpha_{x}\right), y \in U_{T}^{\in}\left(A ; \alpha_{y}\right)$, $a * b \in U_{I}^{\epsilon}\left(A ; \beta_{a}\right), b \in U_{I}^{\epsilon}\left(A ; \beta_{b}\right), u * v \in U_{F}^{\in}\left(A ; \gamma_{u}\right)$, and $v \in U_{F}^{\in}\left(A ; \gamma_{v}\right)$ for all $\alpha_{x}, \alpha_{y}, \beta_{a}, \beta_{b} \in(0,1]$ 
and $\gamma_{u}, \gamma_{v} \in[0,1)$. Then $A_{T}(x * y) \geq \alpha_{x}, A_{T}(y) \geq \alpha_{y}, A_{I}(a * b) \geq \beta_{a}, A_{I}(b) \geq \beta_{b}, A_{F}(u * v) \leq \gamma_{u}$, and $A_{F}(v) \leq \gamma_{v}$. It follows from Equation (20) that

$$
\begin{aligned}
& A_{T}(x) \geq A_{T}(x * y) \wedge A_{T}(y) \geq \alpha_{x} \wedge \alpha_{y}, \\
& A_{I}(a) \geq A_{I}(a * b) \wedge A_{I}(b) \geq \beta_{a} \wedge \beta_{b}, \\
& A_{F}(u) \leq A_{F}(u * v) \vee A_{F}(v) \leq \gamma_{u} \vee \gamma_{v} .
\end{aligned}
$$

Hence $x \in U_{T}^{\in}\left(A ; \alpha_{x} \wedge \alpha_{y}\right), a \in U_{I}^{\in}\left(A ; \beta_{a} \wedge \beta_{b}\right)$, and $u \in U_{F}^{\in}\left(A ; \gamma_{u} \vee \gamma_{v}\right)$. Therefore $A=\left(A_{T}, A_{I}\right.$, $\left.A_{F}\right)$ is an $(\in, \in)$-neutrosophic ideal of $X$.

Conversely, let $A=\left(A_{T}, A_{I}, A_{F}\right)$ be an $(\epsilon, \in)$-neutrosophic ideal of $X$. If there exists $x_{0} \in X$ such that $A_{T}(0)<A_{T}\left(x_{0}\right)$, then $x_{0} \in U_{T}^{\epsilon}(A ; \alpha)$ and $0 \notin U_{T}^{\epsilon}(A ; \alpha)$, where $\alpha=A_{T}\left(x_{0}\right)$. This is a contradiction, and thus $A_{T}(0) \geq A_{T}(x)$ for all $x \in X$. Assume that $A_{T}\left(x_{0}\right)<A_{T}\left(x_{0} * y_{0}\right) \wedge A_{T}\left(y_{0}\right)$ for some $x_{0}, y_{0} \in X$. Taking $\alpha:=A_{T}\left(x_{0} * y_{0}\right) \wedge A_{T}\left(y_{0}\right)$ implies that $x_{0} * y_{0} \in U_{T}^{\in}(A ; \alpha)$ and $y_{0} \in U_{T}^{\in}(A ; \alpha)$; but $x_{0} \notin U_{T}^{\in}(A ; \alpha)$. This is a contradiction, and thus $A_{T}(x) \geq A_{T}(x * y) \wedge A_{T}(y)$ for all $x, y \in X$. Similarly, we can verify that $A_{I}(0) \geq A_{I}(x) \geq A_{I}(x * y) \wedge A_{I}(y)$ for all $x, y \in X$. Now, suppose that $A_{F}(0)>A_{F}(a)$ for some $a \in X$. Then $a \in U_{F}^{\epsilon}(A ; \gamma)$ and $0 \notin U_{F}^{\epsilon}(A ; \gamma)$ by taking $\gamma=A_{F}(a)$. This is impossible, and thus $A_{F}(0) \leq A_{F}(x)$ for all $x \in X$. Suppose there exist $a_{0}, b_{0} \in X$ such that $A_{F}\left(a_{0}\right)>A_{F}\left(a_{0} * b_{0}\right) \vee A_{F}\left(b_{0}\right)$, and take $\gamma:=A_{F}\left(a_{0} * b_{0}\right) \vee A_{F}\left(b_{0}\right)$. Then $a_{0} * b_{0} \in U_{F}^{\in}(A ; \gamma)$, $b_{0} \in U_{F}^{\in}(A ; \gamma)$, and $a_{0} \notin U_{F}^{\in}(A ; \gamma)$, which is a contradiction. Thus $A_{F}(x) \leq A_{F}(x * y) \vee A_{F}(y)$ for all $x, y \in X$. Therefore $A=\left(A_{T}, A_{I}, A_{F}\right)$ satisfies Equation (20).

Lemma 2. Every $(\in, \in)$-neutrosophic ideal $A=\left(A_{T}, A_{I}, A_{F}\right)$ of a $B C K / B C I$-algebra $X$ satisfies

$$
(\forall x, y \in X)\left(x \leq y \Rightarrow A_{T}(x) \geq A_{T}(y), A_{I}(x) \geq A_{I}(y), A_{F}(x) \leq A_{F}(y)\right) .
$$

Proof. Let $x, y \in X$ be such that $x \leq y$. Then $x * y=0$, and thus

$$
\begin{aligned}
& A_{T}(x) \geq A_{T}(x * y) \wedge A_{T}(y)=A_{T}(0) \wedge A_{T}(y)=A_{T}(y), \\
& A_{I}(x) \geq A_{I}(x * y) \wedge A_{I}(y)=A_{I}(0) \wedge A_{I}(y)=A_{I}(y), \\
& A_{F}(x) \leq A_{F}(x * y) \vee A_{F}(y)=A_{F}(0) \vee A_{F}(y)=A_{F}(y),
\end{aligned}
$$

by Equation (20). This completes the proof.

Theorem 8. A NS $A=\left(A_{T}, A_{I}, A_{F}\right)$ in a $B C K$-algebra $X$ is an $(\epsilon, \epsilon)$-neutrosophic ideal of $X$ if and only if $A=\left(A_{T}, A_{I}, A_{F}\right)$ satisfies

$$
(\forall x, y, z \in X)\left(x * y \leq z \Rightarrow\left\{\begin{array}{l}
A_{T}(x) \geq A_{T}(y) \wedge A_{T}(z) \\
A_{I}(x) \geq A_{I}(y) \wedge A_{I}(z) \\
A_{F}(x) \leq A_{F}(y) \vee A_{F}(z)
\end{array}\right)\right.
$$

Proof. Let $A=\left(A_{T}, A_{I}, A_{F}\right)$ be an $(\in, \in)$-neutrosophic ideal of $X$, and let $x, y, z \in X$ be such that $x * y \leq z$. Using Theorem 7 and Lemma 2, we have

$$
\begin{aligned}
& A_{T}(x) \geq A_{T}(x * y) \wedge A_{T}(y) \geq A_{T}(y) \wedge A_{T}(z) \\
& A_{I}(x) \geq A_{I}(x * y) \wedge A_{I}(y) \geq A_{I}(y) \wedge A_{I}(z) \\
& A_{F}(x) \leq A_{F}(x * y) \vee A_{F}(y) \leq A_{F}(y) \vee A_{F}(z)
\end{aligned}
$$


Conversely, assume that $A=\left(A_{T}, A_{I}, A_{F}\right)$ satisfies Equation (22). Because $0 * x \leq x$ for all $x \in X$, it follows from Equation (22) that

$$
\begin{aligned}
& A_{T}(0) \geq A_{T}(x) \wedge A_{T}(x)=A_{T}(x), \\
& A_{I}(0) \geq A_{I}(x) \wedge A_{I}(x)=A_{I}(x), \\
& A_{F}(0) \leq A_{F}(x) \vee A_{F}(x)=A_{F}(x),
\end{aligned}
$$

for all $x \in X$. Because $x *(x * y) \leq y$ for all $x, y \in X$, we have

$$
\begin{aligned}
& A_{T}(x) \geq A_{T}(x * y) \wedge A_{T}(y) \\
& A_{I}(x) \geq A_{I}(x * y) \wedge A_{I}(y) \\
& A_{F}(x) \leq A_{F}(x * y) \vee A_{F}(y)
\end{aligned}
$$

for all $x, y \in X$ by Equation (22). It follows from Theorem 7 that $A=\left(A_{T}, A_{I}, A_{F}\right)$ is an $(\in, \in)$-neutrosophic ideal of $X$.

Theorem 9. If $A=\left(A_{T}, A_{I}, A_{F}\right)$ is an $(\in, \in)$-neutrosophic ideal of a $B C K / B C I$-algebra $X$, then the lower neutrosophic $\in_{\Phi}$-subsets of $X$ are I-energetic subsets of $X$ where $\Phi \in\{T, I, F\}$.

Proof. Let $x, a, u \in X, \alpha, \beta \in(0,1]$, and $\gamma \in[0,1)$ be such that $x \in L_{T}^{\in}(A ; \alpha), a \in L_{I}^{\in}(A ; \beta)$, and $u \in L_{F}^{\in}(A ; \gamma)$. Using Theorem 7 , we have

$$
\begin{aligned}
& \alpha \geq A_{T}(x) \geq A_{T}(x * y) \wedge A_{T}(y), \\
& \beta \geq A_{I}(a) \geq A_{I}(a * b) \wedge A_{I}(b), \\
& \gamma \leq A_{F}(u) \leq A_{F}(u * v) \vee A_{F}(v),
\end{aligned}
$$

for all $y, b, v \in X$. It follows that

$$
\begin{aligned}
& A_{T}(x * y) \leq \alpha \text { or } A_{T}(y) \leq \alpha, \text { that is, } x * y \in L_{T}^{\epsilon}(A ; \alpha) \text { or } y \in L_{T}^{\in}(A ; \alpha) ; \\
& A_{I}(a * b) \leq \beta \text { or } A_{I}(b) \leq \beta, \text { that is, } a * b \in L_{T}^{\epsilon}(A ; \beta) \text { or } b \in L_{T}^{\epsilon}(A ; \beta) ;
\end{aligned}
$$

and

$$
A_{F}(u * v) \geq \gamma \text { or } A_{F}(v) \geq \gamma, \text { that is, } u * v \in L_{T}^{\in}(A ; \gamma) \text { or } v \in L_{T}^{\in}(A ; \gamma) .
$$

Hence $\{y, x * y\} \cap L_{T}^{\epsilon}(A ; \alpha),\{b, a * b\} \cap L_{I}^{\epsilon}(A ; \beta)$, and $\{v, u * v\} \cap L_{F}^{\in}(A ; \gamma)$ are nonempty, and therefore $L_{T}^{\in}(A ; \alpha), L_{I}^{\in}(A ; \beta)$ and $L_{F}^{\in}(A ; \gamma)$ are $I$-energetic subsets of $X$.

Corollary 2. If $A=\left(A_{T}, A_{I}, A_{F}\right)$ is an $(\in, \in)$-neutrosophic ideal of a BCK/BCI-algebra $X$, then the strong lower neutrosophic $\in_{\Phi}$-subsets of $X$ are I-energetic subsets of $X$ where $\Phi \in\{T, I, F\}$.

Proof. Straightforward.

Theorem 10. Let $(\alpha, \beta, \gamma) \in \Lambda_{T} \times \Lambda_{I} \times \Lambda_{F}$, where $\Lambda_{T}, \Lambda_{I}$, and $\Lambda_{F}$ are subsets of $[0,1]$. If $A=\left(A_{T}, A_{I}\right.$, $\left.A_{F}\right)$ is an $(\in, \in)$-neutrosophic ideal of a BCK-algebra $X$, then

(1) the (strong) upper neutrosophic $\in_{\Phi}$-subsets of $X$ are right stable where $\Phi \in\{T, I, F\}$;

(2) the (strong) lower neutrosophic $\in_{\Phi}$-subsets of $X$ are right vanished where $\Phi \in\{T, I, F\}$.

Proof. (1) Let $x \in X, a \in U_{T}^{\epsilon}(A ; \alpha), b \in U_{I}^{\epsilon}(A ; \beta)$, and $c \in U_{F}^{\in}(A ; \gamma)$. Then $A_{T}(a) \geq \alpha, A_{I}(b) \geq \beta$, and $A_{F}(c) \leq \gamma$. Because $a * x \leq a, b * x \leq b$, and $c * x \leq c$, it follows from Lemma 2 that $A_{T}(a *$ $x) \geq A_{T}(a) \geq \alpha, A_{I}(b * x) \geq A_{I}(b) \geq \beta$, and $A_{F}(c * x) \leq A_{F}(c) \leq \gamma$; that is, $a * x \in U_{T}^{\in}(A ; \alpha)$, 
$b * x \in U_{I}^{\epsilon}(A ; \beta)$, and $c * x \in U_{F}^{\epsilon}(A ; \gamma)$. Hence the upper neutrosophic $\epsilon_{\Phi^{-s u b s e t s}}$ of $X$ are right stable where $\Phi \in\{T, I, F\}$. Similarly, the strong upper neutrosophic $\in_{\Phi}$-subsets of $X$ are right stable where $\Phi \in\{T, I, F\}$.

(2) Assume that $x * y \in L_{T}^{\epsilon}(A ; \alpha), a * b \in L_{I}^{\epsilon}(A ; \beta)$, and $c * d \in L_{F}^{\epsilon}(A ; \gamma)$ for any $x, y, a, b, c, d \in X$. Then $A_{T}(x * y) \leq \alpha, A_{I}(a * b) \leq \beta$, and $A_{F}(c * d) \geq \gamma$. Because $x * y \leq x, a * b \leq a$, and $c * d \leq c$, it follows from Lemma 2 that $\alpha \geq A_{T}(x * y) \geq A_{T}(x), \beta \geq A_{I}(a * b) \geq A_{I}(a)$, and $\gamma \leq A_{F}(c * d) \leq A_{F}(c)$; that is, $x \in L_{T}^{\in}(A ; \alpha), a \in L_{I}^{\in}(A ; \beta)$, and $c \in L_{F}^{\in}(A ; \gamma)$. Therefore the lower neutrosophic $\in_{\Phi}$-subsets of $X$ are right vanished where $\Phi \in\{T, I, F\}$. In a similar way, we know that the strong lower neutrosophic $\in_{\Phi}$-subsets of $X$ are right vanished where $\Phi \in\{T, I, F\}$.

Definition 5. Let $A=\left(A_{T}, A_{I}, A_{F}\right)$ be a NS in a BCK/BCI-algebra $X$ and $(\alpha, \beta, \gamma) \in \Lambda_{T} \times \Lambda_{I} \times \Lambda_{F}$, where $\Lambda_{T}, \Lambda_{I}$, and $\Lambda_{F}$ are subsets of $[0,1]$. Then $(\alpha, \beta, \gamma)$ is called a neutrosophic permeable I-value for $A=\left(A_{T}, A_{I}, A_{F}\right)$ if the following assertion is valid:

$$
(\forall x, y \in X)\left(\begin{array}{l}
x \in U_{T}^{\in}(A ; \alpha) \Rightarrow A_{T}(x * y) \vee A_{T}(y) \geq \alpha, \\
x \in U_{I}^{\epsilon}(A ; \beta) \Rightarrow A_{I}(x * y) \vee A_{I}(y) \geq \beta, \\
x \in U_{F}^{\epsilon}(A ; \gamma) \Rightarrow A_{F}(x * y) \wedge A_{F}(y) \leq \gamma
\end{array}\right) .
$$

Example 4. (1) In Example 2, $(\alpha, \beta, \gamma)$ is a neutrosophic permeable I-value for $A=\left(A_{T}, A_{I}, A_{F}\right)$.

(2) Consider a BCI-algebra $X=\{0,1, a, b, c\}$ with the binary operation $*$ that is given in Table 7 (see [14]).

Table 7. Cayley table for the binary operation " $*$ ".

\begin{tabular}{llllll}
\hline$*$ & $\mathbf{0}$ & $\mathbf{1}$ & $\mathbf{a}$ & $\mathbf{b}$ & $\mathbf{c}$ \\
\hline 0 & 0 & 0 & $a$ & $b$ & $c$ \\
1 & 1 & 0 & $a$ & $b$ & $c$ \\
$a$ & $a$ & $a$ & 0 & $c$ & $b$ \\
$b$ & $b$ & $b$ & $c$ & 0 & $a$ \\
$c$ & $c$ & $c$ & $b$ & $a$ & 0 \\
\hline
\end{tabular}

Let $A=\left(A_{T}, A_{I}, A_{F}\right)$ be a NS in $X$ that is given in Table 8 .

Table 8. Tabulation representation of $A=\left(A_{T}, A_{I}, A_{F}\right)$.

\begin{tabular}{cccc}
\hline $\boldsymbol{X}$ & $A_{T}(x)$ & $A_{I}(x)$ & $A_{F}(x)$ \\
\hline 0 & 0.33 & 0.38 & 0.77 \\
1 & 0.44 & 0.48 & 0.66 \\
$a$ & 0.55 & 0.68 & 0.44 \\
$b$ & 0.66 & 0.58 & 0.44 \\
$c$ & 0.66 & 0.68 & 0.55 \\
\hline
\end{tabular}

It is routine to check that $(\alpha, \beta, \gamma) \in(0.33,1] \times(0.38,1] \times[0,0.77)$ is a neutrosophic permeable I-value for $A=\left(A_{T}, A_{I}, A_{F}\right)$.

Lemma 3. If a NS $A=\left(A_{T}, A_{I}, A_{F}\right)$ in a $B C K / B C I$-algebra $X$ satisfies the condition of Equation (14), then

$$
(\forall x \in X)\left(A_{T}(0) \leq A_{T}(x), A_{I}(0) \leq A_{I}(x), A_{F}(0) \geq A_{F}(x)\right) .
$$

Proof. Straightforward.

Theorem 11. If a NS $A=\left(A_{T}, A_{I}, A_{F}\right)$ in a BCK-algebra $X$ satisfies the condition of Equation (14), then every neutrosophic permeable I-value for $A=\left(A_{T}, A_{I}, A_{F}\right)$ is a neutrosophic permeable S-value for $A=\left(A_{T}, A_{I}, A_{F}\right)$. 
Proof. Let $(\alpha, \beta, \gamma)$ be a neutrosophic permeable $I$-value for $A=\left(A_{T}, A_{I}, A_{F}\right)$. Let $x, y, a, b, u, v \in X$ be such that $x * y \in U_{T}^{\in}(A ; \alpha), a * b \in U_{I}^{\in}(A ; \beta)$, and $u * v \in U_{F}^{\in}(A ; \gamma)$. It follows from Equations (23), (3), (III), and (V) and Lemma 3 that

$$
\begin{aligned}
\alpha & \leq A_{T}((x * y) * x) \vee A_{T}(x)=A_{T}((x * x) * y) \vee A_{T}(x) \\
& =A_{T}(0 * y) \vee A_{T}(x)=A_{T}(0) \vee A_{T}(x)=A_{T}(x) \\
\beta & \leq A_{I}((a * b) * a) \vee A_{I}(a)=A_{I}((a * a) * b) \vee A_{I}(a) \\
& =A_{I}(0 * b) \vee A_{I}(a)=A_{I}(0) \vee A_{I}(a)=A_{I}(a)
\end{aligned}
$$

and

$$
\begin{aligned}
\gamma & \geq A_{F}((u * v) * u) \wedge A_{F}(u)=A_{F}((u * u) * v) \wedge A_{F}(u) \\
& =A_{F}(0 * v) \wedge A_{F}(u)=A_{F}(0) \wedge A_{F}(u)=A_{F}(u) .
\end{aligned}
$$

Hence $A_{T}(x) \vee A_{T}(y) \geq A_{T}(x) \geq \alpha, A_{I}(a) \vee A_{I}(b) \geq A_{I}(a) \geq \beta$, and $A_{F}(u) \wedge A_{F}(v) \leq A_{F}(u) \leq \gamma$. Therefore $(\alpha, \beta, \gamma)$ is a neutrosophic permeable $S$-value for $A=\left(A_{T}, A_{I}, A_{F}\right)$.

Given a NS $A=\left(A_{T}, A_{I}, A_{F}\right)$ in a $B C K / B C I$-algebra $X$, any upper neutrosophic $\in_{\Phi}$-subsets of $X$ may not be $I$-energetic where $\Phi \in\{T, I, F\}$, as seen in the following example.

Example 5. Consider a $B C K$-algebra $X=\{0,1,2,3,4\}$ with the binary operation $*$ that is given in Table 9 (see [14]).

Table 9. Cayley table for the binary operation "*”.

\begin{tabular}{llllll}
\hline * & $\mathbf{0}$ & $\mathbf{1}$ & $\mathbf{2}$ & $\mathbf{3}$ & $\mathbf{4}$ \\
\hline 0 & 0 & 0 & 0 & 0 & 0 \\
1 & 1 & 0 & 0 & 0 & 0 \\
2 & 2 & 1 & 0 & 1 & 0 \\
3 & 3 & 1 & 1 & 0 & 0 \\
4 & 4 & 2 & 1 & 2 & 0 \\
\hline
\end{tabular}

Let $A=\left(A_{T}, A_{I}, A_{F}\right)$ be a NS in $X$ that is given in Table 10.

Table 10. Tabulation representation of $A=\left(A_{T}, A_{I}, A_{F}\right)$.

\begin{tabular}{cccc}
\hline $\boldsymbol{X}$ & $\boldsymbol{A}_{\boldsymbol{T}}(\boldsymbol{x})$ & $\boldsymbol{A}_{\boldsymbol{I}}(\boldsymbol{x})$ & $\boldsymbol{A}_{\boldsymbol{F}}(\boldsymbol{x})$ \\
\hline 0 & 0.75 & 0.73 & 0.34 \\
1 & 0.53 & 0.45 & 0.58 \\
2 & 0.67 & 0.86 & 0.34 \\
3 & 0.53 & 0.56 & 0.58 \\
4 & 0.46 & 0.56 & 0.66 \\
\hline
\end{tabular}

Then $U_{T}^{\in}(A ; 0.6)=\{0,2\}, U_{I}^{\in}(A ; 0.7)=\{0,2\}$, and $U_{F}^{\in}(A ; 0.4)=\{0,2\}$. Because $2 \in\{0,2\}$ and $\{1,2 * 1\} \cap\{0,2\}=\varnothing$, we know that $\{0,2\}$ is not an I-energetic subset of $X$.

We now provide conditions for the upper neutrosophic $\epsilon_{\Phi^{-}}$-subsets to be $I$-energetic where $\Phi \in\{T, I, F\}$.

Theorem 12. Let $A=\left(A_{T}, A_{I}, A_{F}\right)$ be a NS in a $B C K / B C I$-algebra $X$ and $(\alpha, \beta, \gamma) \in \Lambda_{T} \times \Lambda_{I} \times \Lambda_{F}$, where $\Lambda_{T}, \Lambda_{I}$, and $\Lambda_{F}$ are subsets of $[0,1]$. If $(\alpha, \beta, \gamma)$ is a neutrosophic permeable I-value for $A=\left(A_{T}, A_{I}\right.$,

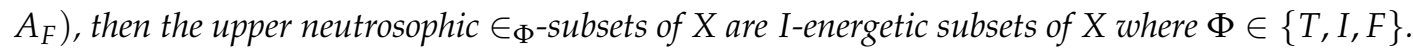


Proof. Let $x, a, u \in X$ and $(\alpha, \beta, \gamma) \in \Lambda_{T} \times \Lambda_{I} \times \Lambda_{F}$, where $\Lambda_{T}, \Lambda_{I}$, and $\Lambda_{F}$ are subsets of $[0,1]$ such that $x \in U_{T}^{\epsilon}(A ; \alpha), a \in U_{I}^{\epsilon}(A ; \beta)$, and $u \in U_{F}^{\epsilon}(A ; \gamma)$. Because $(\alpha, \beta, \gamma)$ is a neutrosophic permeable I-value for $A=\left(A_{T}, A_{I}, A_{F}\right)$, it follows from Equation (23) that

$$
A_{T}(x * y) \vee A_{T}(y) \geq \alpha, A_{I}(a * b) \vee A_{I}(b) \geq \beta, \text { and } A_{F}(u * v) \wedge A_{F}(v) \leq \gamma
$$

for all $y, b, v \in X$. Hence

$$
\begin{aligned}
& A_{T}(x * y) \geq \alpha \text { or } A_{T}(y) \geq \alpha, \text { that is, } x * y \in U_{T}^{\in}(A ; \alpha) \text { or } y \in U_{T}^{\in}(A ; \alpha) ; \\
& A_{I}(a * b) \geq \beta \text { or } A_{I}(b) \geq \beta, \text { that is, } a * b \in U_{I}^{\epsilon}(A ; \beta) \text { or } b \in U_{I}^{\epsilon}(A ; \beta)
\end{aligned}
$$

and

$$
A_{F}(u * v) \leq \gamma \text { or } A_{F}(v) \leq \gamma, \text { that is, } u * v \in U_{F}^{\epsilon}(A ; \gamma) \text { or } v \in U_{F}^{\in}(A ; \gamma)
$$

Hence $\{y, x * y\} \cap U_{T}^{\in}(A ; \alpha),\{b, a * b\} \cap U_{I}^{\epsilon}(A ; \beta)$, and $\{v, u * v\} \cap U_{F}^{\in}(A ; \gamma)$ are nonempty, and therefore the upper neutrosophic $\epsilon_{\Phi}$-subsets of $X$ are $I$-energetic subsets of $X$ where $\Phi \in\{T, I, F\}$.

Theorem 13. Let $A=\left(A_{T}, A_{I}, A_{F}\right)$ be a NS in a BCK/BCI-algebra $X$ and $(\alpha, \beta, \gamma) \in \Lambda_{T} \times \Lambda_{I} \times \Lambda_{F}$, where $\Lambda_{T}, \Lambda_{I}$, and $\Lambda_{F}$ are subsets of $[0,1]$. If $A=\left(A_{T}, A_{I}, A_{F}\right)$ satisfies the following condition:

$$
(\forall x, y \in X)\left(\begin{array}{l}
A_{T}(x) \leq A_{T}(x * y) \vee A_{T}(y) \\
A_{I}(x) \leq A_{I}(x * y) \vee A_{I}(y) \\
A_{F}(x) \geq A_{F}(x * y) \wedge A_{F}(y)
\end{array}\right),
$$

then $(\alpha, \beta, \gamma)$ is a neutrosophic permeable I-value for $A=\left(A_{T}, A_{I}, A_{F}\right)$.

Proof. Let $x, a, u \in X$ and $(\alpha, \beta, \gamma) \in \Lambda_{T} \times \Lambda_{I} \times \Lambda_{F}$, where $\Lambda_{T}, \Lambda_{I}$, and $\Lambda_{F}$ are subsets of $[0,1]$ such that $x \in U_{T}^{\in}(A ; \alpha), a \in U_{I}^{\in}(A ; \beta)$, and $u \in U_{F}^{\in}(A ; \gamma)$. Using Equation (25), we obtain

$$
\begin{aligned}
& \alpha \leq A_{T}(x) \leq A_{T}(x * y) \vee A_{T}(y), \\
& \beta \leq A_{I}(a) \leq A_{I}(a * b) \vee A_{I}(b), \\
& \gamma \geq A_{F}(u) \geq A_{F}(u * v) \wedge A_{F}(v),
\end{aligned}
$$

for all $y, b, v \in X$. Therefore $(\alpha, \beta, \gamma)$ is a neutrosophic permeable $I$-value for $A=\left(A_{T}, A_{I}, A_{F}\right)$.

Combining Theorems 12 and 13, we have the following corollary.

Corollary 3. Let $A=\left(A_{T}, A_{I}, A_{F}\right)$ be a NS in a BCK/BCI-algebra $X$ and $(\alpha, \beta, \gamma) \in \Lambda_{T} \times \Lambda_{I} \times \Lambda_{F}$, where $\Lambda_{T}, \Lambda_{I}$, and $\Lambda_{F}$ are subsets of $[0,1]$. If $A=\left(A_{T}, A_{I}, A_{F}\right)$ satisfies the condition of Equation (25),

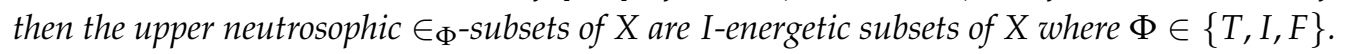

Definition 6. Let $A=\left(A_{T}, A_{I}, A_{F}\right)$ be a NS in a BCK/BCI-algebra $X$ and $(\alpha, \beta, \gamma) \in \Lambda_{T} \times \Lambda_{I} \times \Lambda_{F}$, where $\Lambda_{T}, \Lambda_{I}$, and $\Lambda_{F}$ are subsets of $[0,1]$. Then $(\alpha, \beta, \gamma)$ is called a neutrosophic anti-permeable I-value for $A=\left(A_{T}, A_{I}, A_{F}\right)$ if the following assertion is valid:

$$
(\forall x, y \in X)\left(\begin{array}{c}
x \in L_{T}^{\in}(A ; \alpha) \Rightarrow A_{T}(x * y) \wedge A_{T}(y) \leq \alpha, \\
x \in L_{I}^{\epsilon}(A ; \beta) \Rightarrow A_{I}(x * y) \wedge A_{I}(y) \leq \beta, \\
x \in L_{F}^{\epsilon}(A ; \gamma) \Rightarrow A_{F}(x * y) \vee A_{F}(y) \geq \gamma
\end{array}\right) .
$$


Theorem 14. Let $A=\left(A_{T}, A_{I}, A_{F}\right)$ be a NS in a BCK/BCI-algebra $X$ and $(\alpha, \beta, \gamma) \in \Lambda_{T} \times \Lambda_{I} \times \Lambda_{F}$, where $\Lambda_{T}, \Lambda_{I}$, and $\Lambda_{F}$ are subsets of $[0,1]$. If $A=\left(A_{T}, A_{I}, A_{F}\right)$ satisfies the condition of Equation (19), then $(\alpha, \beta, \gamma)$ is a neutrosophic anti-permeable I-value for $A=\left(A_{T}, A_{I}, A_{F}\right)$.

Proof. Let $x, a, u \in X$ be such that $x \in L_{T}^{\in}(A ; \alpha), a \in L_{I}^{\in}(A ; \beta)$, and $u \in L_{F}^{\in}(A ; \gamma)$. Then

$$
\begin{aligned}
& A_{T}(x * y) \wedge A_{T}(y) \leq A_{T}(x) \leq \alpha, \\
& A_{I}(a * b) \wedge A_{I}(b) \leq A_{I}(a) \leq \beta, \\
& A_{F}(u * v) \vee A_{F}(v) \geq A_{F}(u) \geq \gamma,
\end{aligned}
$$

for all $y, b, v \in X$ by Equation (20). Hence $(\alpha, \beta, \gamma)$ is a neutrosophic anti-permeable $I$-value for $A=\left(A_{T}, A_{I}, A_{F}\right)$.

Theorem 15. Let $A=\left(A_{T}, A_{I}, A_{F}\right)$ be a NS in a BCK/BCI-algebra X and $(\alpha, \beta, \gamma) \in \Lambda_{T} \times \Lambda_{I} \times \Lambda_{F}$, where $\Lambda_{T}, \Lambda_{I}$, and $\Lambda_{F}$ are subsets of $[0,1]$. If $(\alpha, \beta, \gamma)$ is a neutrosophic anti-permeable I-value for $A=\left(A_{T}\right.$, $\left.A_{I}, A_{F}\right)$, then the lower neutrosophic $\in_{\Phi}$-subsets of $X$ are I-energetic where $\Phi \in\{T, I, F\}$.

Proof. Let $x \in L_{T}^{\in}(A ; \alpha), a \in L_{I}^{\in}(A ; \beta)$, and $u \in L_{F}^{\in}(A ; \gamma)$. Then $A_{T}(x * y) \wedge A_{T}(y) \leq \alpha, A_{I}(a * b) \wedge$ $A_{I}(b) \leq \beta$, and $A_{F}(u * v) \vee A_{F}(v) \geq \gamma$ for all $y, b, v \in X$ by Equation (26). It follows that

$$
\begin{aligned}
& A_{T}(x * y) \leq \alpha \text { or } A_{T}(y) \leq \alpha, \text { that is, } x * y \in L_{T}^{\in}(A ; \alpha) \text { or } y \in L_{T}^{\in}(A ; \alpha) \\
& A_{I}(a * b) \leq \beta \text { or } A_{I}(b) \leq \beta, \text { that is, } a * b \in L_{I}^{\in}(A ; \beta) \text { or } b \in L_{I}^{\in}(A ; \beta)
\end{aligned}
$$

and

$$
A_{F}(u * v) \geq \gamma \text { or } A_{F}(v) \geq \gamma, \text { that is, } u * v \in L_{F}^{\in}(A ; \gamma) \text { or } v \in L_{F}^{\in}(A ; \gamma)
$$

Hence $\{y, x * y\} \cap L_{T}^{\in}(A ; \alpha),\{b, a * b\} \cap L_{I}^{\in}(A ; \beta)$ and $\{v, u * v\} \cap L_{F}^{\in}(A ; \gamma)$ are nonempty, and therefore the lower neutrosophic $\in_{\Phi}$-subsets of $X$ are $I$-energetic where $\Phi \in\{T, I, F\}$.

Combining Theorems 14 and 15, we obtain the following corollary.

Corollary 4. Let $A=\left(A_{T}, A_{I}, A_{F}\right)$ be a NS in a BCK/BCI-algebra X and $(\alpha, \beta, \gamma) \in \Lambda_{T} \times \Lambda_{I} \times \Lambda_{F}$, where $\Lambda_{T}, \Lambda_{I}$, and $\Lambda_{F}$ are subsets of $[0,1]$. If $A=\left(A_{T}, A_{I}, A_{F}\right)$ satisfies the condition of Equation (19), then the lower neutrosophic $\in_{\Phi}$-subsets of $X$ are I-energetic where $\Phi \in\{T, I, F\}$.

Theorem 16. If $A=\left(A_{T}, A_{I}, A_{F}\right)$ is an $(\in, \in)$-neutrosophic subalgebra of a BCK-algebra $X$, then every neutrosophic anti-permeable I-value for $A=\left(A_{T}, A_{I}, A_{F}\right)$ is a neutrosophic anti-permeable $S$-value for $A=\left(A_{T}, A_{I}, A_{F}\right)$.

Proof. Let $(\alpha, \beta, \gamma)$ be a neutrosophic anti-permeable $I$-value for $A=\left(A_{T}, A_{I}, A_{F}\right)$. Let $x, y, a, b, u, v \in X$ be such that $x * y \in L_{T}^{\in}(A ; \alpha), a * b \in L_{I}^{\in}(A ; \beta)$, and $u * v \in L_{F}^{\in}(A ; \gamma)$. It follows from Equations (26), (3), (III), and (V) and Proposition 1 that

$$
\begin{aligned}
\alpha & \geq A_{T}((x * y) * x) \wedge A_{T}(x)=A_{T}((x * x) * y) \wedge A_{T}(x) \\
& =A_{T}(0 * y) \wedge A_{T}(x)=A_{T}(0) \wedge A_{T}(x)=A_{T}(x), \\
\beta & \geq A_{I}((a * b) * a) \wedge A_{I}(a)=A_{I}((a * a) * b) \wedge A_{I}(a) \\
& =A_{I}(0 * b) \wedge A_{I}(a)=A_{I}(0) \wedge A_{I}(a)=A_{I}(a),
\end{aligned}
$$

and

$$
\begin{aligned}
\gamma & \leq A_{F}((u * v) * u) \vee A_{F}(u)=A_{F}((u * u) * v) \vee A_{F}(u) \\
& =A_{F}(0 * v) \vee A_{F}(u)=A_{F}(0) \vee A_{F}(u)=A_{F}(u)
\end{aligned}
$$


Hence $A_{T}(x) \wedge A_{T}(y) \leq A_{T}(x) \leq \alpha, A_{I}(a) \wedge A_{I}(b) \leq A_{I}(a) \leq \beta$, and $A_{F}(u) \vee A_{F}(v) \geq A_{F}(u) \geq \gamma$. Therefore $(\alpha, \beta, \gamma)$ is a neutrosophic anti-permeable $S$-value for $A=\left(A_{T}, A_{I}, A_{F}\right)$.

\section{Conclusions}

Using the notions of subalgebras and ideals in $B C K / B C I$-algebras, Jun et al. [8] introduced the notions of energetic subsets, right vanished subsets, right stable subsets, and (anti-)permeable values in $B C K / B C I$-algebras, as well as investigated relations between these sets. As a more general platform that extends the concepts of classic and fuzzy sets, intuitionistic fuzzy sets, and interval-valued (intuitionistic) fuzzy sets, the notion of NS theory has been developed by Smarandache (see [1,2]) and has been applied to various parts: pattern recognition, medical diagnosis, decision-making problems, and so on (see [3-6]). In this article, we have introduced the notions of neutrosophic permeable $S$-values, neutrosophic permeable $I$-values, $(\in, \in)$-neutrosophic ideals, neutrosophic anti-permeable $S$-values, and neutrosophic anti-permeable $I$-values, which are motivated by the idea of subalgebras ( $s$-values) and ideals ( $I$-values), and have investigated their properties. We have considered characterizations of $(\epsilon, \in)$-neutrosophic ideals and have discussed conditions for the lower (upper) neutrosophic $\epsilon_{\Phi}$-subsets to be $S$ - and $I$-energetic. We have provided conditions for a triple $(\alpha, \beta, \gamma)$ of numbers to be a neutrosophic (anti-)permeable $S$ - or $I$-value, and have considered conditions for the upper (lower) neutrosophic $\in_{\Phi}$-subsets to be right stable (right vanished) subsets. We have established relations between neutrosophic (anti-)permeable $S$ - and $I$-values.

Author Contributions: Y.B.J. and S.-Z.S. initiated the main idea of this work and wrote the paper. F.S. and H.B. performed the finding of the examples and checking of the contents. All authors conceived and designed the new definitions and results and read and approved the final manuscript for submission.

Funding: This research received no external funding.

Acknowledgments: The authors wish to thank the anonymous reviewers for their valuable suggestions.

Conflicts of Interest: The authors declare no conflict of interest.

\section{References}

1. Smarandache, F. A Unifying Field in Logics: Neutrosophic Logic. Neutrosophy, Neutrosophic Set, Neutrosophic Probability; American Reserch Press: Rehoboth, NM, USA, 1999.

2. Smarandache, F. Neutrosophic set-a generalization of the intuitionistic fuzzy set. Int. J. Pure Appl. Math. 2005, 24, 287-297.

3. Garg, H.; Nancy. Some new biparametric distance measures on single-valued neutrosophic sets with applications to pattern recognition and medical diagnosis. Information 2017, 8, 126. [CrossRef]

4. Garg, H.; Nancy. Non-linear programming method for multi-criteria decision making problems under interval neutrosophic set environment. Appl. Intell. 2017. [CrossRef]

5. Garg, H.; Nancy. Linguistic single-valued neutrosophic prioritized aggregation operators and their applications to multiple-attribute group decision-making. J. Ambient Intell. Humaniz. Comput. 2018. [CrossRef]

6. Nancy; Garg, H. Novel single-valued neutrosophic aggregated operators under Frank norm operation and its application to decision-making process. Int. J. Uncertain. Quantif. 2016, 6, 361-375. [CrossRef]

7. Jun, Y.B. Neutrosophic subalgebras of several types in BCK/BCI-algebras. Ann. Fuzzy Math. Inform. 2017, 14, 75-86.

8. Jun, Y.B.; Ahn, S.S.; Roh, E.H. Energetic subsets and permeable values with applications in BCK/BCI-algebras. Appl. Math. Sci. 2013, 7, 4425-4438. [CrossRef]

9. Jun, Y.B.; Smarandache, F.; Bordbar, H. Neutrosophic $\mathcal{N}$-structures applied to $B C K / B C I$-algebras. Informations 2017, 8, 128. [CrossRef]

10. Jun, Y.B.; Smarandache, F.; Song, S.Z.; Khan, M. Neutrosophic positive implicative $\mathcal{N}$-ideals in $B C K$-algebras. Axioms 2018, 7, 3. [CrossRef]

11. Öztürk, M.A.; Jun, Y.B. Neutrosophic ideals in BCK/BCI-algebras based on neutrosophic points. J. Int. Math. Virtual Inst. 2018, 8, 1-17. 
12. Song, S.Z.; Smarandache, F.; Jun, Y.B. Neutrosophic commutative $\mathcal{N}$-ideals in $B C K$-algebras. Information 2017, 8, 130. [CrossRef]

13. Huang, Y.S. BCI-Algebra; Science Press: Beijing, China, 2006.

14. Meng, J.; Jun, Y.B. BCK-Algebras; Kyungmoon Sa Co.: Seoul, Korea, 1994.

(C) 2018 by the authors. Licensee MDPI, Basel, Switzerland. This article is an open access article distributed under the terms and conditions of the Creative Commons Attribution (CC BY) license (http://creativecommons.org/licenses/by/4.0/). 